\title{
Stark broadening tables for the helium I 492.2 line
}

\section{Application to weakly coupled plasma diagnostics ${ }^{\star}$}

\author{
N. Lara ${ }^{1}$, M. Á. González ${ }^{2}$, and M. A. Gigosos ${ }^{1}$ \\ ${ }^{1}$ Departamento de Óptica, Universidad de Valladolid, 44071 Valladolid, Spain \\ e-mail: gigosos@coyanza.opt.cie.uva.es \\ 2 Departamento de Física Aplicada, Universidad de Valladolid, 44071 Valladolid, Spain \\ e-mail: manuelgd@termo.uva.es
}

Received 28 February 2012 / Accepted 23 April 2012

\begin{abstract}
Context. We studied the Stark broadening of the He I $492.2 \mathrm{~nm}$ line, which is sometimes used for plasma diagnostics or to obtain information on different stellar parameters.

Aims. The final aim of this study is to obtain tables of lines shapes as well as the relationships between different line parameters and the plasma electron density, temperature, or composition. The tables of profiles and the mathematical expressions obtained will be used in plasma diagnostics.

Methods. We performed computer simulations and used a physical model that considers a weakly coupled plasma. Our computer simulations naturally take into account ion dynamical effects, which has permitted us to study the influence on the line shapes of imbalances in the plasma caused by different electron and gas temperatures.

Results. Our computer simulations considered electron densities between $10^{19}$ and $10^{24} \mathrm{~m}^{-3}$, electron temperatures between 5000 and $40000 \mathrm{~K}$, and plasmas of different compositions. The dependences obtained in the simulations for the line width, the ratio of intensities between the allowed and the forbidden components, or the distances between those components' peaks on the plasma conditions are shown and compared with experimental data. Numerical expressions for the line width and for the peak distances against the electron density were obtained from the simulation results and can be applied to obtain the electron density from experimental results. Full line profile tables are also supplied for use in plasma diagnostics.
\end{abstract}

Key words. line: profiles - atomic data - plasmas - atomic processes

\section{Introduction}

We studied the Stark broadening of the He I $492.2 \mathrm{~nm}$ line in a plasma with computer simulations. The results were compared with the available experimental data and with previous theories and approximate calculations. This line appears as a result of the transition between the $1 \mathrm{~s} 4 \mathrm{~d}^{1} \mathrm{D}$ and the $1 \mathrm{~s} 2 \mathrm{p}^{1} \mathrm{P}^{\circ}$ states and shows one allowed component and two forbidden components, the transitions between the $1 \mathrm{~s} 4 \mathrm{p}^{1} \mathrm{P}^{\circ}$ and the $1 \mathrm{~s} 2 \mathrm{p}^{1} \mathrm{P}^{\circ}$, and between the $1 \mathrm{~s} 4 \mathrm{f}^{1} \mathrm{~F}^{\circ}$ and the $1 \mathrm{~s} 2 \mathrm{p}{ }^{1} \mathrm{P}^{\circ}$ states. Owing to the quasi degenerate set of upper states of the transition, the line shape strongly depends on the density of charged particles in a plasma, therefore the analysis of this dependence can be useful for plasma diagnostics.

Two characteristics of the line are especially relevant for obtaining the electron density, $N_{\mathrm{e}}$, of the plasma: the distance from the $1 \mathrm{~s} 4 \mathrm{f}^{1} \mathrm{~F}^{\circ}-1 \mathrm{~s} 2 \mathrm{p}{ }^{1} \mathrm{P}^{\circ}$ forbidden component to the allowed one and the intensity ratio between these two line components.

The results of this work are shown mainly as tables of line profiles to obtain the plasma conditions through comparison with experimental data and approximate formula obtained by fitting the simulation results for plasma diagnostics. These are the final aims of this study.

\footnotetext{
* Tables of line profiles are only available at the CDS via anonymous ftp to cdsarc.u-strasbg. fr (130.79.128.5) or via http://cdsarc.u-strasbg.fr/viz-bin/qcat?]/A+A/542/A75
}

The shape analysis of the 492.2 line was used to obtain information on He abundances (Leone \& Lanzafame 1998; Castelli \& Hubrig 2004; Lyubimkov et al. 2004; Catanzaro 2008), He stratification (Catanzaro 2008) or radial velocities (Jeffery et al. 2001). Owing to a lack of available information, some authors were in some cases only able to use line widths obtained from approximate models (Castelli \& Hubrig 2004) instead of using the full profiles for comparison with the experimental data, though several authors already pointed out the better results obtained by comparing the full theoretical and experimental profiles instead of using only the line widths (Leckrone 1971). Other authors using full profiles for comparison with experimental data employed old calculations (Heasley \& Wolff 1981; Jeffery et al. 2001) to include the influence of Stark broadening in the profiles. The discrepancies in those and other comparisons neccessitated more advances in line broadening calculations of the He I $492.2 \mathrm{~nm}$ line (Lyubimkov et al. 2004; Castelli \& Hubrig 2004).

In this work we only considered the broadening and shifting of the spectral line caused by the Stark effect. Other effects such as Doppler broadening or van der Waals broadening, which are noticeable at very low electron densities, will not be taken into account. The electron density, the free electrons and ions temperatures and the ions mass will be used as the parameters that characterize the state of the plasma. Our treatment permits us to consider non-equilibrium conditions in which the 
plasma ions and electrons have different temperatures and the calculation technique includes the so-called "ion dynamics effects" in a natural way.

This work completes an earlier publication dedicated to the He I $447.1 \mathrm{~nm}$ line (Gigosos \& González 2009) that has similar characteristics. In that work the special properties of these two spectral lines (492.2 and 447.1), which make them very suitable for plasma diagnostics, were already given, and will not be repeated here. Then, after a brief reminder of the calculation method, the dependences of several line parameters against electron temperature and density are shown and compared with previous experimental data.

\section{Stark broadening calculations}

Our calculations were obtained following the same technique as described in Gigosos \& González (2009). Therefore we only recall some details here and refer the interested reader to that work.

Following Anderson (1949), the Stark broadening of the spectral lines emitted by a plasma is calculated considering the electric field generated by the charged particles, electrons and ions, in the plasma. Under the usual approximations (Gigosos et al. 2003; Gigosos \& González 2009) the emitters in the plasma are considered as statistically independent particles that produce incoherent emissions, and the plasma is considered as a thermal bath whose evolution is not affected by the emission processes. Then, the line profile calculations are performed considering a typical emitter subjected to the electric fields of the charges in the plasma and averaging the results over the possible configurations of perturbers in the plasma and over the emitter states.

As in previous works, the emission profile is obtained as the Fourier transform of the autocorrelation function of the emitter dipole moment:

$$
\begin{aligned}
I(\Delta \omega) & =\operatorname{Re} \frac{1}{\pi} \int_{0}^{\infty} \mathrm{d} t\{C(t)\} \mathrm{e}^{\mathrm{i} \Delta \omega t}, \\
C(t) & =\operatorname{tr}[\boldsymbol{D}(t) \cdot \boldsymbol{D}(0) \rho], \\
\boldsymbol{D}(t) & =U^{+}(t) \boldsymbol{D}(0) U(t),
\end{aligned}
$$

where $U(t)$ is the emitter time evolution operator, which is obtained in the calculation by solving the evolution equation

$\mathrm{i} \hbar \frac{\mathrm{d}}{\mathrm{d} t} U(t)=H(t) U(t)=\left(H_{0}+q \boldsymbol{E}(t) \cdot \boldsymbol{R}\right) U(t)$,

which includes the unperturbed emitter structure through the hamiltonian $H_{0}$, and the plasma perturbation through the dipole interaction with the electric field, produced by the plasma charges on the emitter.

As discussed in Gigosos \& González (2009), only a few emitter states need to be considered in Eq. (4) to obtain the line profiles with good accuracy. We took two groups of atomic states into account, an upper group with 16 states and a lower group with 3 states. The upper states include the $1 \mathrm{~s} 4 \mathrm{~d}^{1} \mathrm{D}$ states, source of the dipole allowed $492.19 \mathrm{~nm}$ transition, and the $1 \mathrm{~s} 4 \mathrm{p}^{1} \mathrm{P}^{\circ}$ and $1 \mathrm{~s} 4 \mathrm{f}^{1} \mathrm{~F}^{\circ}$, origin of the 491.08 and $492.06 \mathrm{~nm}$ dipole forbidden transitions together with the $1 \mathrm{~s} 4 \mathrm{~s}{ }^{1} \mathrm{~S}$ state. These last states are coupled to the $1 \mathrm{~s} 4 \mathrm{~d}^{1} \mathrm{D}$ through the plasma electric field, giving rise to the Stark broadening. The lower group consists of only the $1 \mathrm{~s} 2 \mathrm{p}{ }^{1} \mathrm{P}^{\circ}$ states, which is destination of all the aforementioned transitions, as can be seen in Fig. 1. Because the distance in energies between the upper and lower group of states is considered to be very large compared with the typical energies of the plasma perturbations, the evolution of both sets of states are

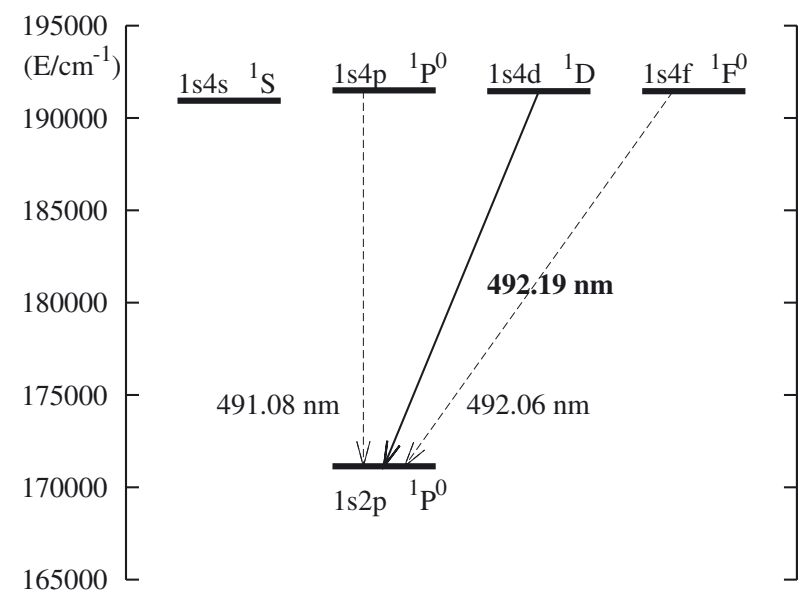

Fig. 1. Diagram of the levels involved in the transition studied in this work.

Table 1. Data of the helium I atomic structure used in this work.

\begin{tabular}{lcccc}
\hline \hline Config. & Term & $E_{i}$ & $E_{k}$ & $S_{i k}$ \\
\hline $1 \mathrm{~s} 4 \mathrm{~s}-1 \mathrm{~s} 4 \mathrm{p}$ & ${ }^{1} \mathrm{~S}-1 \mathrm{P}^{\mathrm{o}}$ & 190940.23 & 191492.71 & $5.112 \mathrm{e}+02$ \\
$1 \mathrm{~s} 4 \mathrm{p}-1 \mathrm{~s} 4 \mathrm{~d}$ & ${ }^{1} \mathrm{P}^{\mathrm{o}}-{ }^{1} \mathrm{D}$ & 191492.71 & 191446.46 & $8.507 \mathrm{e}+02$ \\
$1 \mathrm{~s} 4 \mathrm{~d}-1 \mathrm{~s} 4 \mathrm{f}$ & ${ }^{1} \mathrm{D}-{ }^{1} \mathrm{~F}^{\mathrm{o}}$ & 191446.46 & 191451.90 & $4.828 \mathrm{e}+02$ \\
\hline
\end{tabular}

Notes. The energies $E_{i}$ and $E_{k}$ are given in $\mathrm{cm}^{-1}$ and $S_{i k}$ in atomic units. These data were taken from http://physics.nist.gov/ PhysRefData/ASD/lines_form.html.

obtained independently. Table 1 shows the atomic data relevant to the upper group of states whose coupling through the plasma electric field gives rise to the line broadening and to the appearance of the forbidden components.

As described in Gigosos \& González (2009), our calculations were performed with computer simulations that reproduce the electric field on the emitter in a plasma. Inserting this electric field into Eqs. (1)-(4) permits one to obtain the broadened line profile corresponding to a single plasma configuration. The final profile is obtained after averaging over many (typically over 30000 samples in this work) plasma configurations. Therefore, the final profile is calculated from the averaged autocorrelation $\{C(t)\}$ obtained considering the autocorrelations corresponding to each field sequence.

Our computer simulation technique is a molecular dynamics technique with independent particles that uses the so-called $\mu$-ion model (Seidel \& Stamm 1982). More details have been given elsewhere (Gigosos et al. 2003; Gigosos \& González 2009). This technique is valid only for lowly coupled plasmas. Accordingly, the cases of plasma electron density, $N_{\mathrm{e}}$, and temperature, $T_{\mathrm{e}}$, considered in these calculations which correspond to high values of the coupling parameter must be considered as extrapolations of this method (see Dufour et al. 2005, for a discussion of this approximation) and serve only as an illustration of the results for different conditions.

\section{Results}

\subsection{Shape of the spectral line}

As mentioned before, to obtain different plasma conditions one usually compares the experimental measurements to calculated line profiles. Accodingly, we begin discussing our results by comparing full profiles obtained in the simulations with those from previous calculations as well as experimental results. 


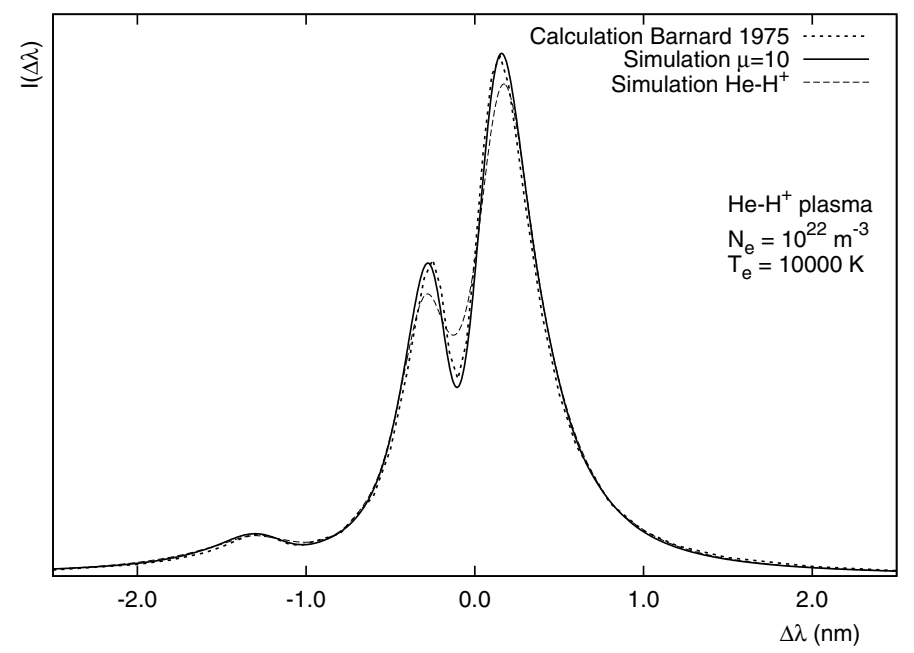

Fig. 2. Comparison of a previous theoretical calcualtion (Barnard et al. 1975) with two simulation calculations. Ion dynamics effects are noticeable in the centre of the line.

Figure 2 shows the comparison of two simulation calculations with a previous analytical result (Barnard et al. 1975). Though Barnard et al. (1975) used a calculation that included ion dynamical effects, for densities higher than $10^{22} \mathrm{~m}^{-3}$ the calculation considered a static theory. Figure 2 compares their profile at $N_{\mathrm{e}}=10^{22} \mathrm{~m}^{-3}$ and $T_{\mathrm{e}}=10000 \mathrm{~K}$ with two simulation results, one for perturbers with a high reduced mass (or low ionic temperature) whose behaviour would be close to static perturbers and another with $\mathrm{H}^{+}$perturbers. Clearly, although the overall shape of the line is very similar for the three calculations, ion dynamics effects still affect the central part of the profile. While the theoretical static calculation and the high reduced mass simulation result agree, the profile obtained for full dynamic perturbers shows much less structure in the centre of the line.

\subsection{Ion dynamics effects}

Ion dynamics effects are also shown in Fig. 3. Here simulated profiles are compared with the experimental measurements in Richter \& Piel (1985), performed for different plasma compositions with $\mathrm{H}^{+}, \mathrm{He}^{+}$or $\mathrm{Ar}^{+}$as perturbers to study ion dynamics effects in the line. In Richter \& Piel (1985), plasma density was obtained with interferometry and for the profiles in the figure it was established to be $N_{\mathrm{e}}=10^{21} \mathrm{~m}^{-3}, T_{\mathrm{e}}$ through relative line intensities and from line to continuum ratio and gas temperature using Doppler broadening. The simulated profiles in Fig. 3 are those that agree best with the experimental ones. These comparisons show that computer simulations reproduce ion dynamic effects well. But it is also important to notice that the conditions of these calculations correspond to values of electron density and temperature similar to those obtained independently in the experiment.

\subsection{General comparison with experimental data}

Another comparison with previous calculations (Barnard et al. 1975; Schöning 1994) and experiment (Richter \& Piel 1985) is shown in Fig. 4. Here the simulated profile was obtained for the values of $N_{\mathrm{e}}$ and $T_{\mathrm{e}}$ given in the experiment. Simulations and experiment again agree well.

Figure 5 shows a last comparison between experimental profiles and simulations. The experimental data in this figure

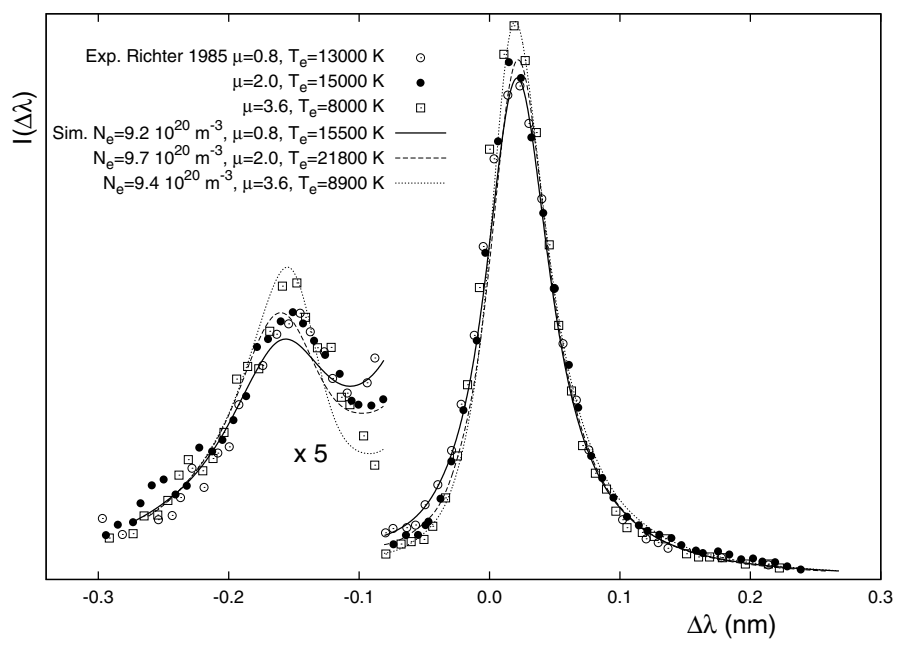

Fig. 3. Comparison of experimental (Richter \& Piel 1985) and simulated profiles to study the influence of ion dynamics effects on the line profile. The measured electronic density is $10^{21} \mathrm{~m}^{-3}$.

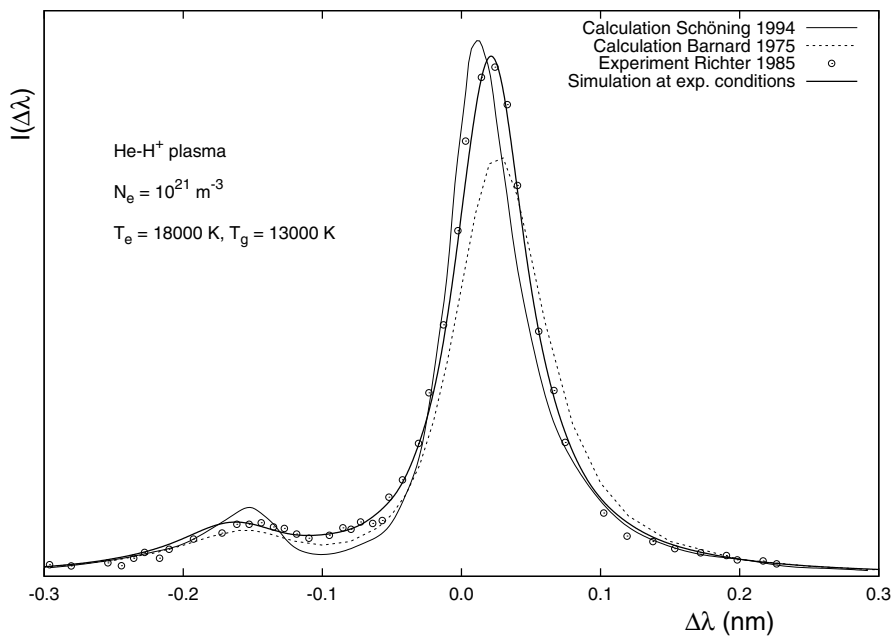

Fig. 4. Comparison of different theoretical profiles (Barnard et al. 1975; Schöning 1994) with computer simulations and an experimental result from Richter \& Piel (1985).

correspond to measurements at four different radial positions (Birkeland et al. 1971), so that a dependence of the line shape with the electron density can be observed in the figure. Radial distribution of the electron density in (Birkeland et al. 1971) was determined from the Stark broadening of three He I lines and from the integrated intensity of the He I $501.6 \mathrm{~nm}$ line. Close but not equal values were obtained through the different lines. The ranges of the experimentally determined electron densities are represented with vertical dashed lines in the square within Fig. 5 for the four radial positions corresponding to the profiles in the figure. The simulated profiles shown in the figure correspond to those that agree best with the experimental ones. Evidently, computer simulations satisfactorily reproduce the change in the shape of the line as the electron density increases. But is more interesting for diagnostics purposes that the electron densities corresponding to these calculated profiles are well within the ranges determined experimentally for each radial position. As can be seen in the inserted square, these values of electron density, which are represented with black full squares, fall near the centre of the experimentally determined range of $N_{\mathrm{e}}$ for most of the cases. 


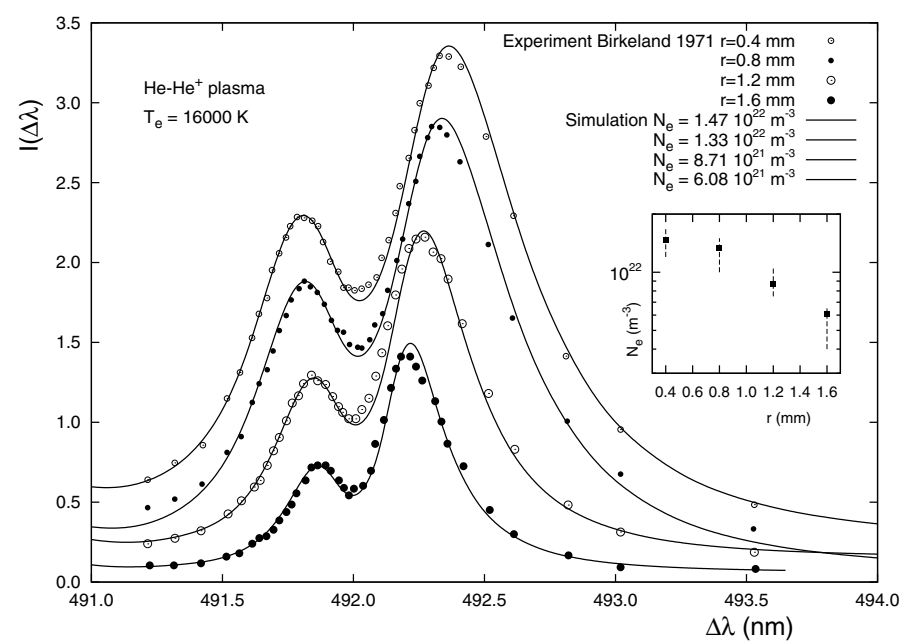

Fig. 5. Comparison between experimental and simulated full line profiles for different conditions of the plasma electron density corresponding to four radial positions in the experiment. The square within the figure shows with dashed lines the range of electron densities obtained from different methods in the experiment, while the squared point corresponds to the electron density of the calculated profile for each position.

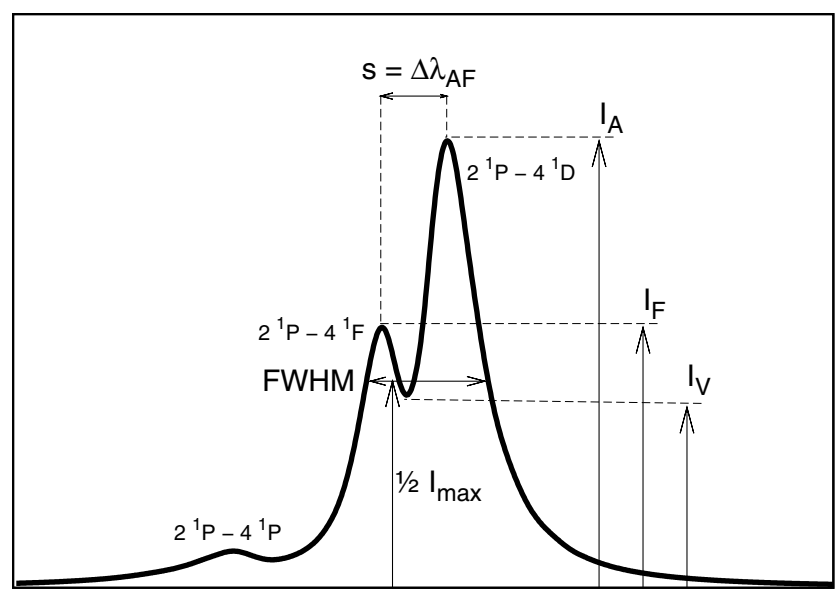

Fig. 6. Definition of the line parameters.

\subsection{FWHM and electronic impact broadening}

Now we study the behaviour of different line parameters to analyse if they are suitable in plasma diagnostics. The parameters studied here are the full width at half maximum (FWHM) the ratio of the forbidden component to the allowed one, $I_{\mathrm{F}} / I_{\mathrm{A}}$, the ratio of the central valley intensity to the allowed peak intensity, $I_{\mathrm{D}} / I_{\mathrm{A}}$, and the distance between the allowed and forbidden peaks, $s$. These parameters are illustrated in Fig. 6 with a profile obtained in the simulation.

The dependence of the line FWHM versus the electron density, $N_{\mathrm{e}}$, is shown in Fig. 7. The results obtained in two types of simulations are shown in this figure. The first are simulations considering a full plasma, ions and electrons, for a pure $\mathrm{He}$ plasma in thermodynamical equilibrium with $T_{\mathrm{e}}=T_{\mathrm{gas}}$, which corresponds to a value of the reduced mass $\mu=2$. Evidently, the FWHM shows a small step upwards around $N_{\mathrm{e}}=10^{22} \mathrm{~m}^{-3}$, which approximately corresponds to the density at which the intensity of the forbidden component reaches one half of the allowed one, so that the FWHM now includes the width of both components, while at lower densities only the allowed component width contributes to the FWHM. The second set of

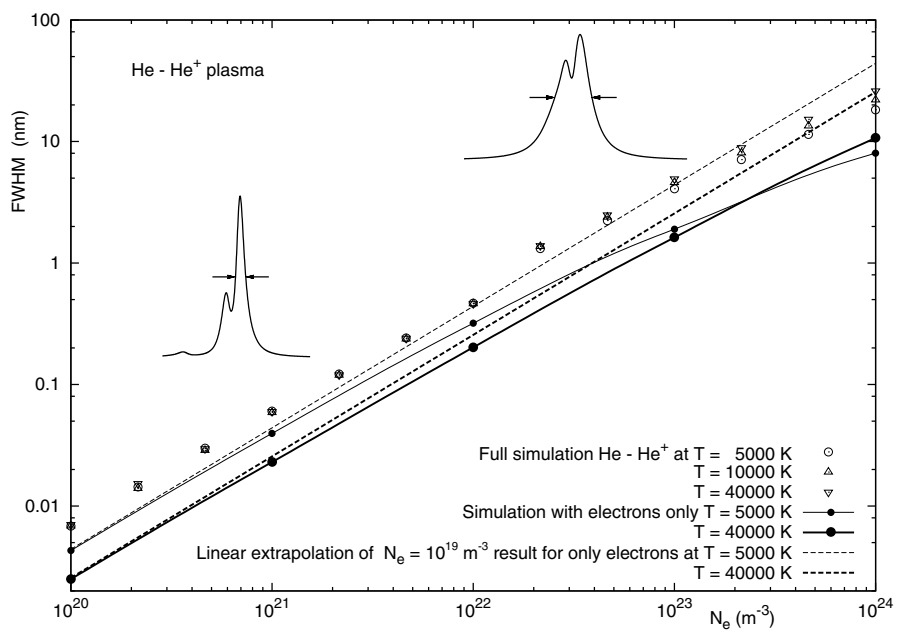

Fig. 7. Dependence of the FWHM on the electron density for a full plasma simulation considering ions and electrons in the case of pure He plasma and for an one-component plasma considering only electrons. Different temperatures were considered in both cases. Two dashed lines with the unity slope corresponding to the electronic broadening at low densities illustrate the behaviour predicted by the impact approximation. The difference between the pure electronic plasma results and the impact estimation illustrates the departure of the impact behaviour.

simulations are one-component plasma simulations, OCP, considering only electrons. These were performed to obtain the electronic impact broadening and to analyse the range of validity of the impact approximation.

Two different electron temperatures were considered in these OCP simulations to study the influence of $T_{\mathrm{e}}$ in the impact behaviour. For the lower values of $N_{\mathrm{e}}$ considered in the simulations (which were performed down to electron densities of $10^{19} \mathrm{~m}^{-3}$, beyond the limits of the figure) the electronic width shows a linear dependence on $N_{\mathrm{e}}$, which proves the validity of the impact approximation, but as the electron density increases, the electronic width behaviour starts to depart from that linear trend. Of course, the lower the temperature, the more noticeable this departure will be. Two dashed straight lines with unity slope corresponding to the electronic broadening at the lowest densities are represented to better illustrate the inaccuracy of impact approximation at high densities.

Table 2 shows a comparison of the simulation results for the OCP calculation with those obtained using standard theoretical models considering only the broadening caused by the electronic impact. The results for the FWHM are very close for the three different calculations while the shift shows higher discrepancies. Simulation results were obtained through a calculation for $N_{\mathrm{e}}=$ $10^{19} \mathrm{~m}^{-3}$ and were extrapolated to the density of reference $N_{\mathrm{e}}=$ $10^{22} \mathrm{~m}^{-3}$ (the extrapolation lines are represented with dashed lines in Fig. 7).

It is very useful to have simple expressions obtained by fitting the relationship between the electronic density and the line width obtained in the simulations. At low densities $\left(N_{\mathrm{e}}<\right.$ $10^{22} \mathrm{~m}^{-3}$ ), when the measured width corresponds only to the allowed component (see Fig. 7), the simulation results fit quite well to a function of the type

$$
\log _{10}(F W H M)=0.4756\left(\frac{N_{\mathrm{e}}}{10^{22}}\right)^{0.9118} \quad\left(\text { for } N_{\mathrm{e}}<10^{22}\right)
$$

$$
F W H M \text { in } \mathrm{nm}, N_{\mathrm{e}} \text { in } \mathrm{m}^{-3} \text {, }
$$

where the full widths due to both ions and electrons are taken into account and all data corresponding to different temperatures 
Table 2. FWHM and peak shift in $\mathrm{nm}$ for an only electron broadening calculation normalised at $N_{\mathrm{e}}=10^{22} \mathrm{~m}^{-3}$.

\begin{tabular}{l|cc|cc|cc}
\hline \hline$T(\mathrm{~K})$ & \multicolumn{2}{|c|}{ ST } & \multicolumn{2}{c|}{ D \& SB } & \multicolumn{2}{c}{ This work } \\
& $F W H M$ & Shift & $F W H M$ & Shift & $F W H M$ & Shift \\
\hline 5000 & 0.460 & 0.1020 & 0.398 & 0.0983 & 0.440 & 0.0913 \\
10000 & 0.392 & 0.0773 & 0.346 & 0.0642 & 0.380 & 0.0671 \\
20000 & 0.326 & 0.0584 & 0.294 & 0.0370 & 0.320 & 0.0447 \\
40000 & 0.270 & 0.0440 & 0.246 & 0.0241 & 0.256 & 0.0311 \\
\hline
\end{tabular}

Notes. The values of the simulation correspond to a linear extrapolation of the value obtained in simulations at $N_{\mathrm{e}}=10^{19} \mathrm{~m}^{-3}$. (ST: see Griem 1974; D \& SB: see Dimitrijević \& Sahal-Bréchot 1990).

Table 3. Values of the fitting parameters $a$ and $b$ of the fit $\log _{10}(F W H M /(\mathrm{nm}))=a \log _{10}\left(N_{\mathrm{e}} / \mathrm{m}^{-3}\right)+b$ for values of electron density $10^{22}<N_{\mathrm{e}}<10^{24} \mathrm{~m}^{-3}$.

\begin{tabular}{lcc}
\hline \hline$T(\mathrm{~K})$ & $a$ & $b$ \\
\hline 5000 & 0.6928 & -15.3425 \\
10000 & 0.7308 & -16.1701 \\
20000 & 0.7562 & -16.7303 \\
40000 & 0.7703 & -17.0489 \\
\hline
\end{tabular}

for the case $\mu=2$ have been considered jointly. If one requires a more accurate fitting, the expression

$$
\begin{aligned}
\log _{10}(F W H M)= & -0.020253 \cdot\left(\log _{10}\left(N_{\mathrm{e}}\right)\right)^{2} \\
& +1.7624 \cdot\left(\log _{10}\left(N_{\mathrm{e}}\right)\right)-29.305
\end{aligned}
$$

can be used, with the same units as in (5), and for the same electron densities.

When the electron density is higher than $10^{22} \mathrm{~m}^{-3}$ the allowed and forbidden components overlap (see Fig. 7) so that the parameter FWHM includes both components. For these cases, the influence of temperature is noticeable and it must be considered in the fittings. Table 3 gives the values of the fitting parameters to a function of the type

$\log _{10}(F W H M)=a \cdot\left(\log _{10}\left(N_{\mathrm{e}}\right)\right)+b$

for the temperatures considered in this work.

Figure 8 shows a comparison between the calculated and experimental FWHM. Computer simulations correspond to three different temperatures, 5000, 20000 , and $40000 \mathrm{~K}$ to check its influence on the FWHM and to cover the different conditions of the experiments. While the experiments shown in the figure generally agree with the simulations, the two older experiments increasingly disagree as the electron density decreases. Similar discrepancies are found for these two experiments in the comparisons with other parameters shown below.

\subsection{Ratio of allowed and forbidden component intensities}

A line characteristic that can be used to obtain the plasma electron density is the ratio between the most intense forbidden component and the allowed one, $I_{\mathrm{F}} / I_{\mathrm{A}}$. While at low densities the forbidden component is very weak, at medium or high densities it can be easily recorded and the value of $I_{\mathrm{F}} / I_{\mathrm{A}}$ obtained. Figure 9 shows a comparison of this parameter obtained in the simulations and in different experiments covering wide ranges of electron density and temperature. For the simulations three different cases are shown to check the influence of plasma composition or temperature in this parameter: for pure He plasma $(\mu=2)$ at $T_{\mathrm{e}}=T_{\text {gas }}=5000$ and $20000 \mathrm{~K}$ and for a $\mathrm{He}-\mathrm{H}^{+}$plasma $(\mu=0.5)$ with $T_{\mathrm{e}}=T_{\text {gas }}=5000 \mathrm{~K}$. A good agreement is

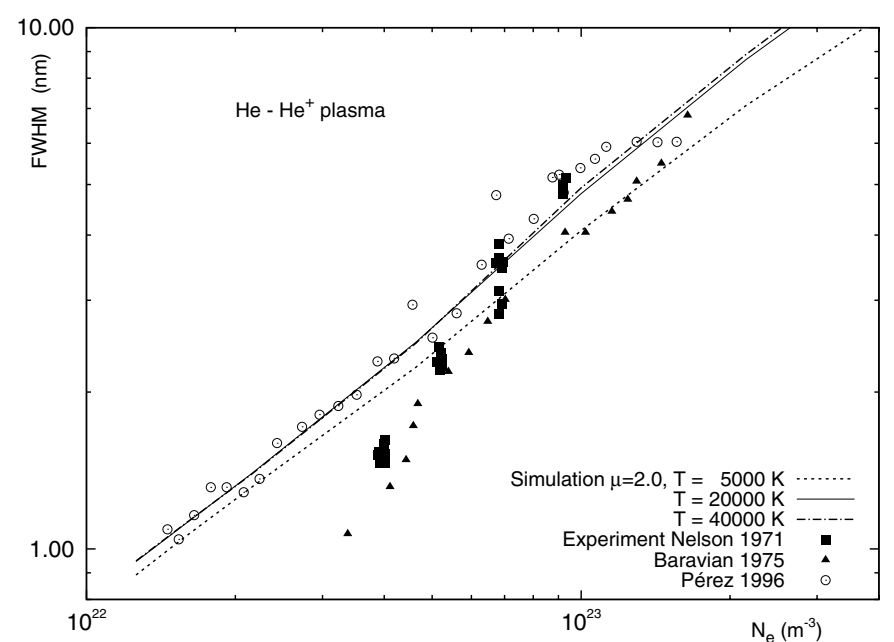

Fig. 8. Comparison of the simulated and measured FWHM.

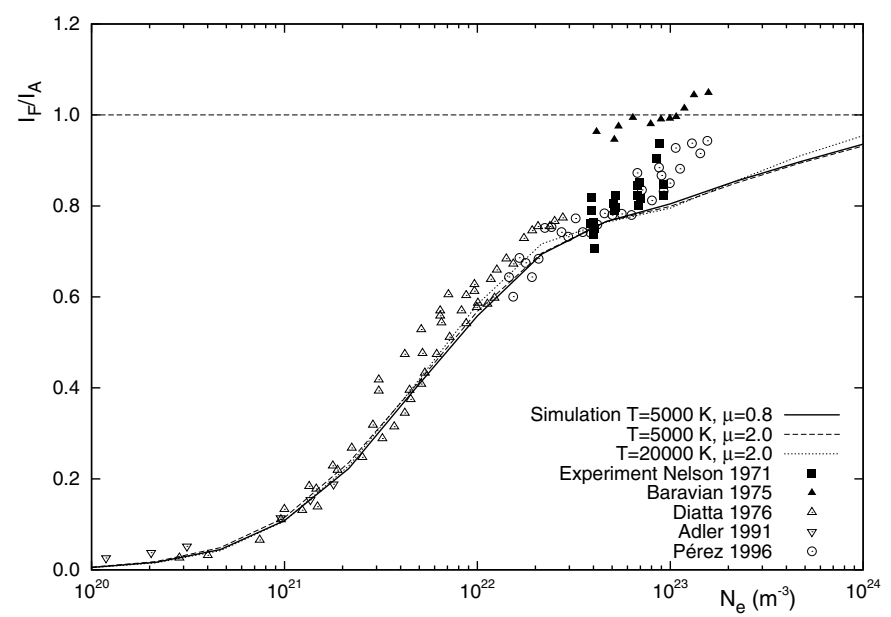

Fig. 9. Comparison experiment-simulation for the ratio of intensities between the forbidden and the allowed line components.

found along the wide range of electron densities shown in the figure in spite of the different conditions of all the experiments shown here. Clearly, the ratio $I_{\mathrm{F}} / I_{\mathrm{A}}$ is quite insensitive to the electron temperature for the ranges shown in the figure, which supports its suitability for determining the electron density value in a plasma.

\subsection{Intensity ratio of the central dip and the allowed component}

Computer simulations also allow us to study plasmas far from equilibrium. In these simulations the situations where the gas temperature are lower than the electrons temperature in the plasma are represented with higher values of the reduced mass $\mu$ than the one that would correspond to the plasma (González \& Gigosos 2009). The existence of this imbalance can affect the line shapes and, in turn, the line parameters used in plasma diagnostics. This is the case of the ratio, $I_{\mathrm{D}} / I_{\mathrm{A}}$, between the intensities of the central dip of the line and the allowed component. Imbalances in gas and electronic temperatures can notably affect the values of $I_{\mathrm{D}} / I_{\mathrm{A}}$, therefore its measurement is no reliable diagnostic for the plasma density. This is shown in Fig. 10 where we compare the experimental measurements (Nelson \& Barnard 1971; Baravian et al. 1975; Diatta et al. 1976; Pérez et al. 1996) and the values of $I_{\mathrm{D}} / I_{\mathrm{A}}$ obtained from the 


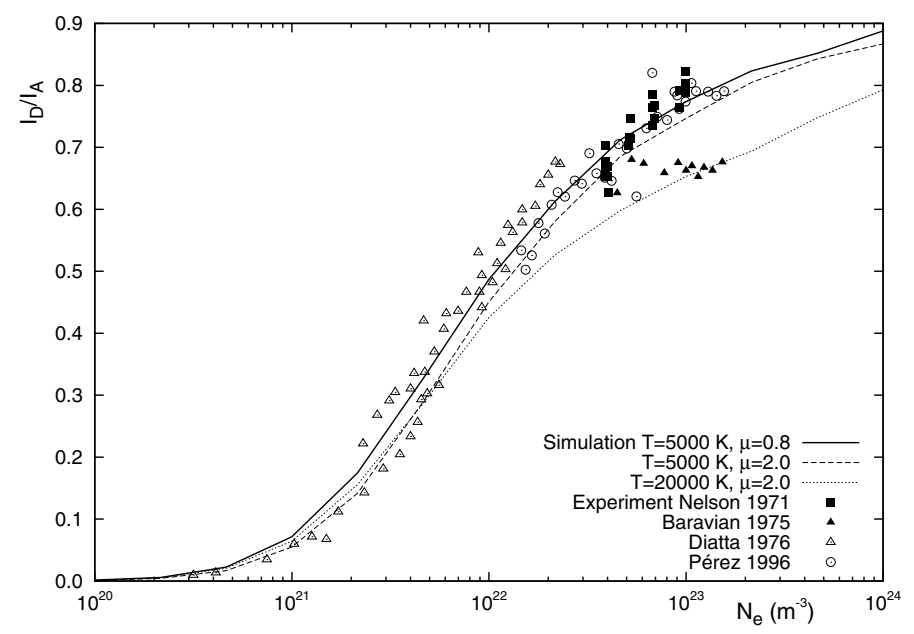

Fig. 10. Comparison between experiments and simulations of the ratio $I_{\mathrm{D}} / I_{\mathrm{A}}$ for different temperatures and values of $\mu$.



Fig. 11. Allowed-forbidden peaks distance and allowed peak shift versus the electron density of the plasma for a pure He plasma. Results for three temperatures are displayed in each case. These parameters values were measured on the simulated full profiles.

simulated profiles. Evidently, experiment and simulation agree well. However, the most interesting point in this figure is the strong dependence of this parameter on the electron temperature at the higher densities considered in the figure, when its measurement is easier, so that, as mentioned before, the measurement of this parameter is no accurate diagnostics method.

\subsection{Distance between the allowed and the forbidden components}

On the other hand, a parameter that can easily be employed in plasma diagnostics is the distance between the allowed and the forbidden components, $s$. In a previous work (Ivković et al. 2010) the dependence of this parameter on the electron density was used to obtain a simple expression that permits one to calculate $N_{\mathrm{e}}$ from an experimental measurement. An equivalent expression has been obtained here for the He I $492.2 \mathrm{~nm}$ line. Figure 11 shows the dependence obtained in the simulations for the distance between the allowed and the forbidden line components and for the shift of the allowed component on the electron density for different temperatures. As can be seen there, the value of $s$ depends on the electron density and temperature, therefore the fitting must take both parameters into account.

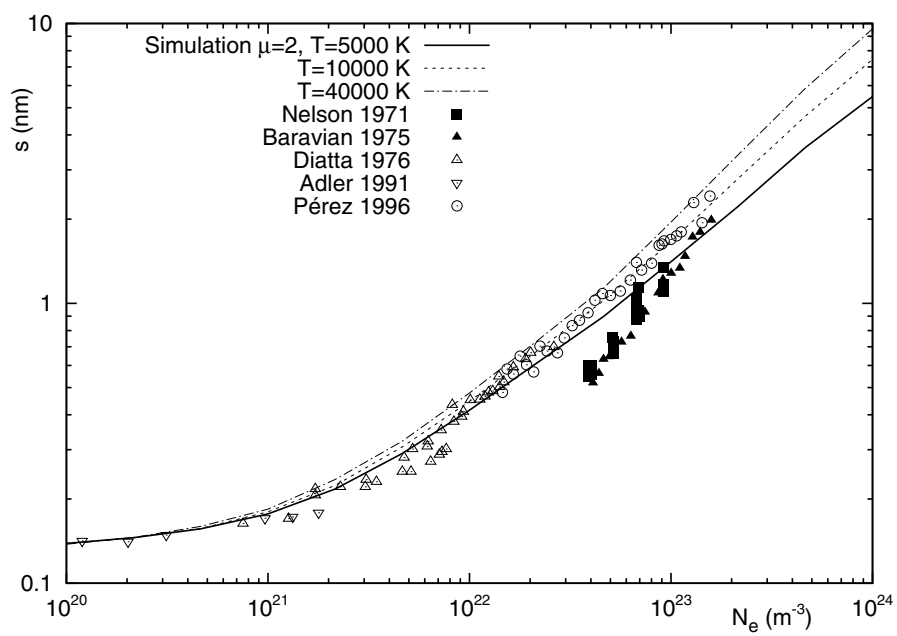

Fig. 12. Comparison of experimental and simulated values of the distance between the allowed and the forbidden peaks of the line. The simulated values were obtained measuring that parameter in the full profiles obtained in the simulations.

However the reduced mass $\mu$ has a minor influence, as also seen in Gigosos \& González (2009) for the $447.1 \mathrm{~nm}$ line, which implies that the same fitting can be used for different plasma compositions and for conditions far from equilibrium when electron and gas temperature are different.

The good agreement between the value of parameter $s$ obtained in the calculations and experimental measurements, shown in Fig. 12, reinforces the reliability of using this parameter as a mean to obtain the electron density in the plasma. As can be seen in that figure, simulation results for a pure He plasma in thermodynamical equilibrium and different electron temperatures have been compared to results from different experiments (Nelson \& Barnard 1971; Baravian et al. 1975; Diatta et al. 1976; Adler \& Piel 1991; Pérez et al. 1996) and a general good agreement is found. As happened before in other comparisons, the worst agreement corresponds to the comparison with the results of Nelson \& Barnard (1971) and Baravian et al. (1975).

To obtain a practical expression for use in plasma diagnostics, we performed a fitting of the simulation results obeying a function of the type

$\left(\frac{s}{s_{0}}\right)^{b}=1+\left(\frac{N}{N_{0}}\right)^{a}$

or, which is the same,

$\log _{10}\left(N_{\mathrm{e}}\right)=\log _{10}\left(N_{0}\right)+\frac{1}{a} \log _{10}\left(\left(\frac{s}{s_{0}}\right)^{b}-1\right)$.

The criterion followed to chose this type of fitting is the same as employed in Ivković et al. (2010), where a more complete description of the details is given. The procedure combines minimal squares and MinMax fittings ${ }^{1}$. The most convenient result is given by the expressions

$$
\begin{aligned}
b(T) & =1.25+\frac{994}{T} \\
\log _{10}\left(N_{\mathrm{e}}\right) & =21.3065+\frac{1}{0.8766} \log _{10}\left(\left(\frac{s}{0.13187}\right)^{b(T)}-1\right)
\end{aligned}
$$

with $N_{\mathrm{e}}$ in $\mathrm{m}^{-3}, T$ in $K$ and $s$ in $\mathrm{nm}$.

\footnotetext{
${ }^{1}$ In that publication there is a printing mistake: the captions of Tables 1 and 2 are interchanged.
} 


\subsection{Tables of line profiles}

The results of this work are given as tables of full line profiles in electronic format. These tables (numbered as Tables 4 to 16) are available via anonymous ftp to cdsarc.u-srasbourg. fr. These tables form a set that covers a range of electron densities between $10^{20}$ and $10^{24} \mathrm{~m}^{-3}$, temperatures between 5000 and $40000 \mathrm{~K}$ and values of the parameter $\mu$, reduced mass of the emitter-perturber pair, between $0.8\left(\mathrm{He}-\mathrm{H}^{+}\right.$plasma in thermodynamic equilibrium) and 10.0, which corresponds to a situation where the gas temperature is much lower than the electronic temperature.

Acknowledgements. This work has been partially financed by Spanish Ministerio de Educación y Ciencia through grant ENE2007-63386/FTN, by the Spanish Ministerio de Ciencia e Innovación under grant ENE2010-19542 and by the Junta de Castilla y León through grant VA007A09.

\section{References}

Adler, G., \& Piel, A. 1991, J. Quant. Spectrosc. Radiat. Transf., 45, 11 Anderson, P. W. 1949, Phys. Rev., 76, 647

Baravian, G., Bretagne, J., Godart, J., \& Sultan, G. 1975, Z. Phys. B, 20, 255

Barnard, A. J., Cooper, J., \& Smith, E. W. 1975, J. Quant. Spectrosc. Radiat. Transf., 15, 429
Birkeland, J. W., Bacon, M. E., \& Braun, W. G. 1971, Phys. Rev. A, 3, 354

Castelli, F., \& Hubrig, S. 2004, A\&A, 425, 263

Catanzaro, G. 2008, MNRAS, 385, L33

Diatta, C. S., Czernichowski, A., \& Chapelle, J. 1976, Physica, 84C, 425

Dimitrijević, M. S., \& Sahal-Bréchot, S. 1990, A\&AS, 82, 519

Dufour, E., Calisti, A., Talin, B., et al. 2005, Phys. Rev. E, 71, 066409

Gigosos, M. A., \& González, M. Á. 2009, A\&A, 503, 293

Gigosos, M. A., González, M. Á., \& Cardeñoso, V. 2003, Spectrochim. Acta Part B, 58, 1489

González, M. Á., \& Gigosos, M. A. 2009, Plasma Sources Sci. Technol., 18, 034001

Griem, H. R. 1974, Spectral Line Broadening by Plasmas (New York: Academic Press)

Heasley, J. N., \& Wolff, S. C. 1981, ApJ, 245, 977

Ivković, M., González, M. Á., Jovićević, S., Gigosos, M. A., \& Konjević, N. 2010, Spectrochim. Acta Part B, 65, 234

Jeffery, C. S., Wolff, V. M., \& Pollacco, D. L. 2001, A\&A, 376, 497

Leckrone, D. S. 1971, A\&A, 11, 387

Leone, F., \& Lanzafame, A. C. 1998, A\&A, 330, 306

Lyubimkov, L. S., Rostopchin, S. I., \& Lambert, D. L. 2004, MNRAS, 351, 745

Nelson, R. H., \& Barnard, A. J. 1971, J. Quant. Spectrosc. Radiat. Transf., 11, 161

Pérez, C., de la Rosa, I., Aparicio, J. A., Mar, S., \& Gigosos, M. A. 1996, Jpn. J. Appl. Phys., 35, 4073

Richter, H., \& Piel, A. 1985, J. Quant. Spectrosc. Radiat. Transf., 33, 615

Schöning, T. 1994, J. Phys. B: At. Mol. Opt. Phys., 27, 4501

Seidel, J., \& Stamm, R. 1982, J. Quant. Spectrosc. Radiat. Transf., 27, 499 